\title{
Proceedings of the 2017 Australian X-ray Analytical Association workshops, conference, and exhibition
}

This special issue of Powder Diffraction offers a selection of contributions from the Australian X-ray Analytical Association (AXAA) workshops, conference, and exhibition held on 5-9th February 2017 (AXAA-2017) in Melbourne, Australia. The AXAA (http://www.axaa.org) is a not-for-profit organisation formed of a diverse group of scientists and technologists employed in mining and manufacturing industries, research and educational organisations, as well as instrument manufacturers throughout Australia, the Asia Pacific, and beyond.

The AXAA holds an event every 3 years as a forum for scientific and technical discussion and information interchange between workers in the fields of X-ray analysis, including X-ray diffraction and fluorescence (XRD and $\mathrm{XRF}$ ), which are the traditional focus areas of AXAA, as well as in small angle X-ray scattering, X-ray absorption spectroscopy, XRF mapping and tomography, and neutron scattering, which form a significant part of the event as Australia's synchrotron and neutron facilities mature. At AXAA-2017, nearly one-quarter of the 200 delegates travelled from outside of Australia, from New Zealand, Canada, USA, Germany, Netherlands, France, Italy, Denmark, Sweden, Finland, Switzerland, Italy, Singapore, Hong Kong, Japan, Mongolia, China, Malaysia, and Pakistan.

The theme for AXAA-2017 was "Innovation from Characterisation", reflecting the current drive of Australia to be an innovation nation. The importance and utility of characterization to innovation in Australia was presented in talks covering a wide range of topics, from Dr. Kathy Ehrig's Plenary presentation on the role of XRF and XRD on BHP's Olympic Dam mining and processing operations, to an invited presentation on biomimetic mineralisation of metal-organic

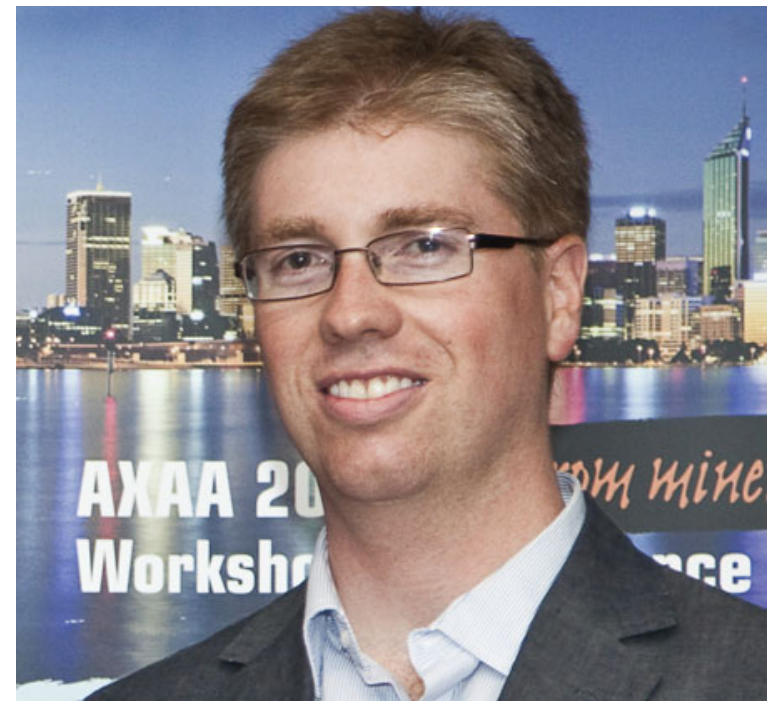

Photo 1: Nathan Webster frameworks at the surface of living cells, and to the talk by a Student Bursary Award recipient on trapping $\mathrm{CO}_{2}$ and toxic metals. Plenary presentations were also given by Dr. Thomas Proffen (Director Neutron Data Analysis and Visualisation at Oak Ridge National Laboratory, USA) "Structural Characterization of Complex Materials", Dr. Bridget Ingham (Senior Scientist at Callaghan Innovation, New Zealand) "Innovation from X-ray Characterisation in Materials and Energy Research", Dr. Chris Ryan (Senior Principal Research Scientist, CSIRO Mineral Resources) "Super HighDefinition Synchrotron XRF Element Imaging: A Catalyst for Innovative Research", and Prof. Andrew Peele (Director, Australian Synchrotron) "Innovation From Characterisation at The Australian Synchrotron".

Continuing on from the success of the inaugural AXAA-2014 Public Lecture, "The Mars Science Laboratory Experience", by Prof. David Bish (Indiana State University), two Public Lectures were given as an exciting opening to AXAA-2017: "Journeying to the Centres of the Planets" by Dr. Helen Maynard-Casely (Planetary and Beamline Scientist, Australian Centre for Neutron Scattering, ANSTO) and "When Art and Science Collide: X-ray Fluorescence Elemental Mapping of Nineteenth Century Paintings From The National Gallery of Victoria" by Michael Varcoe-Cocks (Head of Conservation, National Gallery of Victoria).

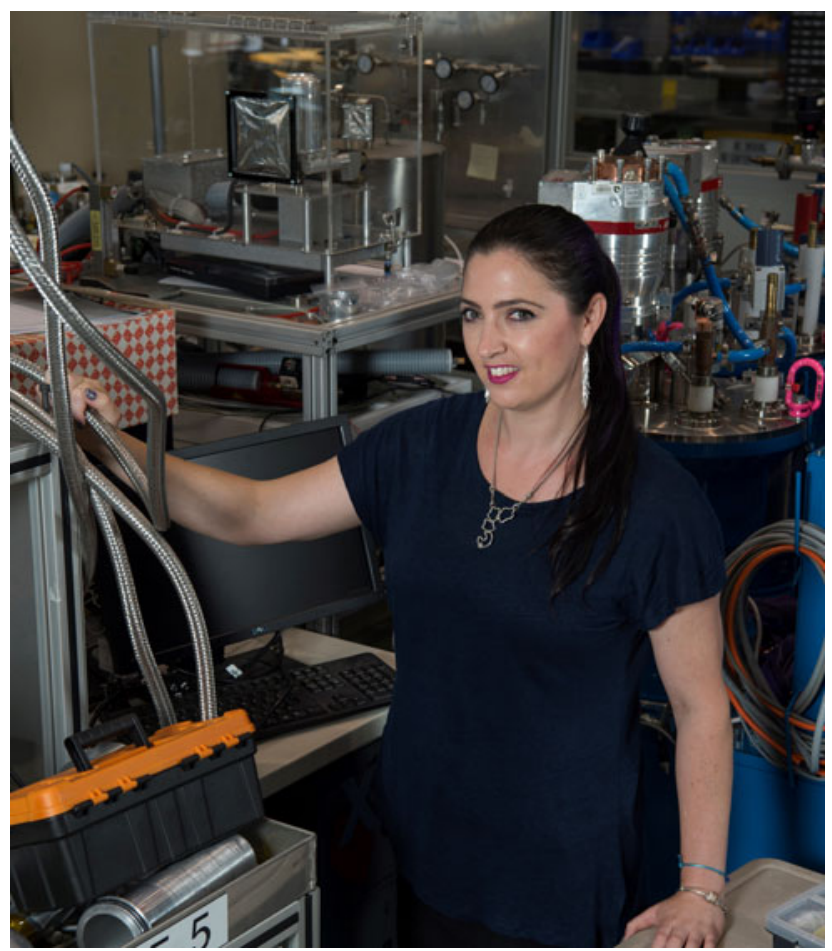

Photo 2: Vanessa Peterson 
A new addition to AXAA-2017 was the Career Path Panel Discussion session, of particular value to students and early career researchers, and live demonstrations showcasing the latest developments in X-ray instrumentation and equipment. Following AXAA-2017 delegates had the opportunity to tour the Australian Synchrotron, Australia's major X-ray characterisation and research facility, as well as the conservation facilities at the National Gallery of Victoria.

Diversity was forefront in the AXAA-2017 conference policy, and in the area of gender diversity, the proportion of female delegates was substantially higher than in previous events, with $43 \%$ of plenary, $38 \%$ of invited, and $53 \%$ of conference committee members female.
AXAA-2017 demonstrated a continued high level of interest and engagement in X-ray and related analytical methods, applications and research in Australia and surrounds. The next AXAA conference event will be in 2020 (AXAA-2020).

The guest editors of this special issue of Powder Diffraction:

Nathan A.S. Webster CSIRO Mineral Resources, Clayton, Australia

Vanessa K. Peterson Australian Centre for Neutron Scattering, ANSTO, Lucas Heights, Australia 